\title{
The effect of multileaf collimator leaf width on the radiosurgery planning for spine lesion treatment in terms of the modulated techniques and target complexity
}

Soo-Min Chae ${ }^{1}$, Gi Woong Lee ${ }^{1}$ and Seok Hyun Son ${ }^{2 *}$

\begin{abstract}
Purpose: We aim to evaluate the effects of multileaf collimator (MLC) leaf width (5 mm vs. $2.5 \mathrm{~mm}$ ) on the radiosurgery planning for the treatment of spine lesions according to the modulated techniques (intensitymodulated radiotherapy [IMRT] vs. volumetric-modulated arc therapy [VMAT]) and the complexity of the target shape.

Methods: For this study, artificial spinal lesions were contoured and used for treatment plans. Three spinal levels (C5, T5, and L2 spines) were selected, and four types of target shapes reflecting the complexity of lesions were contoured. The treatment plans were performed using $2.5-\mathrm{mm}$ and $5-\mathrm{mm}$ MLCs, and also using both static IMRT and VMAT. In total, 48 treatment plans were established. The efficacy of each treatment plan was compared using target volume coverage (TVC), conformity index (Cl), dose gradient index $(\mathrm{Gl})$, and $\mathrm{V}_{30 \%}$.

Results: When the 5-mm MLC was replaced by the $2.5-\mathrm{mm} \mathrm{MLC,} \mathrm{TVC} \mathrm{and} \mathrm{GI} \mathrm{improved} \mathrm{significantly} \mathrm{by} 5.68 \%$ and $6.25 \%$, respectively, while CI did not improve. With a smaller MLC leaf width, the improvement ratios of the TVC were larger in IMRT than VMAT (8.38\% vs. 2.97\%). In addition, the TVC was improved by $14.42-16.74 \%$ in target type 4 compared to the other target types. These improvements were larger in IMRT than in VMAT (27.99\% vs. 6.34\%). The $V_{30 \%}$ was not statistically different between IMRT and VMAT according to the MLC leaf widths and the types of target.
\end{abstract}

Conclusion: The smaller MLC leaf width provided improved target coverage in both IMRT and VMAT, and its improvement was larger in IMRT than in VMAT. In addition, the smaller MLC leaf width was more effective for complex-shaped targets.

Keywords: Multileaf collimator, Intensity-modulated radiotherapy, Volumetric-modulated arc therapy, Target complexity

\section{Background}

Spine metastasis can cause intractable pain, and when it damages the spinal cord, it can lead to motor and/or sensory dysfunction. Consequently, spine metastasis is the primary cause of deterioration in the quality of life of cancer patients $[1,2]$. The treatment of spine metastasis includes surgery, systemic chemotherapy, radiotherapy,

\footnotetext{
* Correspondence: sonshyun@catholic.ac.kr

${ }^{2}$ Department of Radiation Oncology, Incheon St. Mary's hospital, College of Medicine, the Catholic University of Korea, Incheon, Korea

Full list of author information is available at the end of the article
}

as well as medical treatment with analgesics and steroids; most patients undergo radiotherapy. In the past, radiotherapy consisted of delivering modest doses of radiation to spinal lesions using conventional techniques, but through advances in planning and delivery techniques, radiosurgery, which can deliver highly localized doses of radiation, is now being widely used [2-4]. Because spinal lesions are only slightly affected by respiration or internal organ movement, those are suitable for radiosurgery, which is effective in reducing pain [3,5-8]. 
Three-dimensional conformal radiotherapy (3D-CRT), dynamic conformal arc therapy (DCAT), and intensitymodulated radiotherapy (IMRT) are commonly used in radiosurgery. Since the recent development of volumetricmodulated arc therapy (VMAT), in which the IMRT technique is combined with the DCAT technique, several studies have been conducted on it [3,5,9-12]. The multileaf collimator (MLC), which is used for linac-based radiosurgery, continues to be developed, and is now commercially available with a $2.5-\mathrm{mm}$ leaf width. Several studies using 3D-CRT, DCAT, and IMRT have reported that smaller MLC leaf width provides dosimetric improvement, particularly in the radiosurgery for small lesions, and the use of micro-MLC, which has a leaf width of less than $5 \mathrm{~mm}$, has provided good results [13-16]. The differences in VMAT planning related to different MLC leaf width sizes ( $5 \mathrm{~mm}$ vs. $2.5 \mathrm{~mm}$ ) have not been evaluated. Furthermore, the effects of a smaller MLC width have not been evaluated by comparing VMAT with other techniques. The existing researches investigated the effects of MLC leaf width size in relation to the target shape [16-18]. However, these researches were limited because the target complexities were not methodically classified.

In this study, we evaluated the difference in dosimetric effects between 5-mm and 2.5-mm MLC leaf width in radiosurgery of spine lesions. We established the change in the quality of the dose distribution for the modulated techniques (VMAT and IMRT) and verified the effects of MLC leaf width in relation to the complexity of the target shape.

\section{Methods}

\section{Target delineation}

This study was designed to evaluate the dosimetric effects of different MLC leaf widths on the quality of the dose distribution in relation to the radiotherapy techniques and target shape complexity. Therefore, artificial spine lesions were contoured and used for treatment plans.

For the simulations, patients were immobilized using a thermoplastic head mask for $\mathrm{C}$ spine and the BodyFix system (Medical Intelligence, $\mathrm{GmbH}$, Schwabmuenchen, Germany) for T and L spines. Spiral computed tomography $(\mathrm{CT})$ scans were performed using the Ingenuity 128-channel CT scanner (Philips Healthcare, Eindhoven, The Netherlands) with a 1-mm slice thickness. The patients' CT data were retrospectively reviewed and used for this planning study following institutional review board approval (IRB of Incheon St. Mary's Hospital, the Catholic University of Korea, Reference number: OC13RISI0061). Written informed consent was obtained from the patient for the publication of this report and any accompanying images.

To represent various shapes of spinal lesions, three spinal levels (C5, T5, and L2) were selected, and four types of target shapes, reflecting the complexity of the lesions, were contoured (Figure 1). The four types of spinal lesions were contoured by modifying a Weinstein, Boriani, and Biagnini (WBB) surgical staging system, which provides various degrees of spinal lesions surrounding the spinal cord [19-21]. Type 1 is defined as the entire vertebral body only (WBB zones $4,5,6,7,8$, and 9), and type 2 is defined as the vertebral body and left transverse process (WBB zones 4, 5, 6, 7, 8, 9, 10, and 11). Type 3 is defined as the vertebral body, left transverse process, and spinous process (WBB zones 1 , $4,5,6,7,8,9,10,11$, and 12), and type 4 is defined as the vertebral body, left transverse process, spinous process, and right transverse process (entire spinal cord encompassed state, WBB zones 1-12). The spinal cord is defined as the spinal canal minus $1 \mathrm{~mm}$ from the entire circumference, as the gap between the target and the spinal cord was $1 \mathrm{~mm}$. The spinal cord was contoured by extending $10 \mathrm{~mm}$ both above and below the level of the target volume. Relevant structures such as the esophagus, lungs, and both kidneys were also contoured. The target volumes and spinal cord volume in accordance with the level of the spine and the types of target shapes are summarized in Table 1.

\section{Prescription and radiation treatment planning}

The prescribed dose was 18 Gy in a single fraction, with a maximum dose of 10 Gy allowed to $0.25 \mathrm{cc}$ of spinal cord [22]. In this study, we intended to obtain the maximal target volume coverage satisfying the dose constraint for the spinal cord. Therefore, the irradiated dose to $0.25 \mathrm{cc}$ of spinal cord is $10 \mathrm{~Gy}$, which is the same for all types of plans. However, the coverage rates of the prescribed dose to the target volume varied according to each plan.

All treatment plans were performed with Eclipse version 8.9 (Varian Medical Systems, Palo Alto, CA) to exclude any bias due to the effect of different planning algorithms. The Dose Volume Optimizer version 8.9 for IMRT and the Progressive Resolution Optimizer version 8.9 for VMAT were used for the plan optimization. The Anisotropic Analytic Algorithm version 8.9 was used for the dose calculations. The treatment plans were performed using a $2.5-\mathrm{mm}$ and $5-\mathrm{mm}$ MLC and using both multiple static field sliding window IMRT and multi-arc VMAT. For each plan, the same parameters such as the isocenter location; the number of fields; the MLC margin; the gantry, collimator, and couch angles for each beam; and the dose constraints level were used. The IMRT plans consisted of single-isocenter, coplanar, and 11 fields delivered by the sliding-window method (dynamic MLC mode). The fluence map pixel size was $2 \times$ $2 \mathrm{~mm}^{2}$. The whole angles were $0^{\circ}, 33^{\circ}, 65^{\circ}, 98^{\circ}, 130^{\circ}$, $163^{\circ}, 195^{\circ}, 228^{\circ}, 260^{\circ}, 293^{\circ}$, and $326^{\circ}$. The VMAT plans 


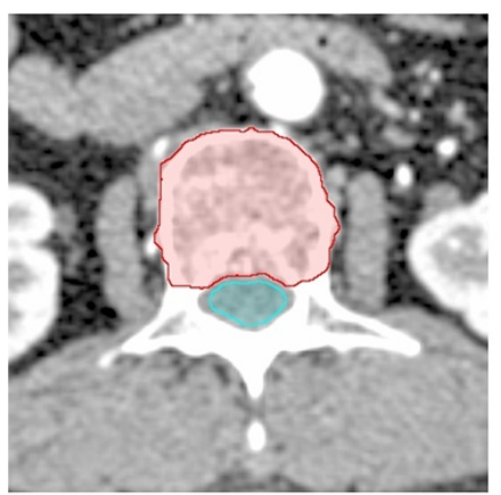

(a) Type 1

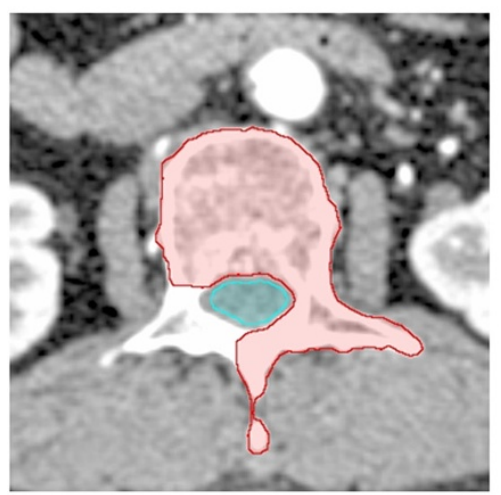

(c) Type 3

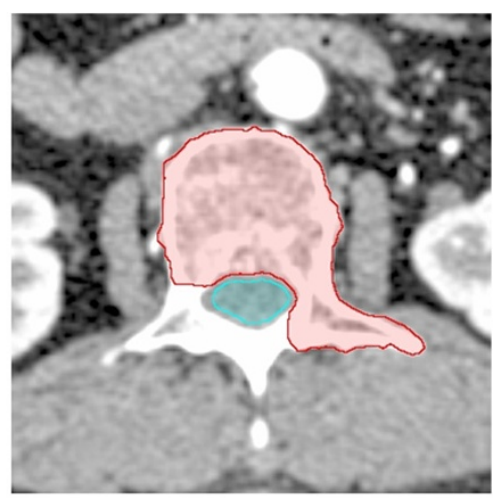

(b) Type 2

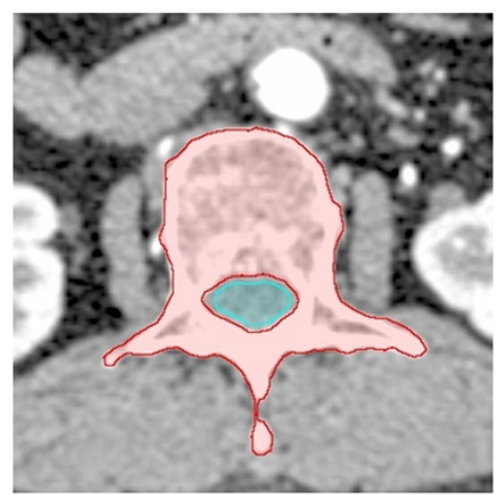

(d) Type 4

Figure 1 Four types of target shapes, reflecting the complexity of lesions. (a) Type 1, (b) Type 2, (c) Type 3, and (d) Type 4.

were implemented with a single-isocenter 3-full arc, and without a couch rotation. The collimator angles were set to $45^{\circ}, 315^{\circ}$, and $90^{\circ}$ for each arc. The gantry angle range of each arc was $179.9^{\circ}-181.1^{\circ}$. Several studies have reported that static IMRT planning using more than 7 fields results in a dosimetric gain $[5,23]$, and that VMAT planning using multi-arc, rather than 1-arc, achieves better results $[3,24]$. Therefore, in this study, for the best results for the two different planning techniques, we use an 11-field static IMRT and a 3-arc VMAT.

Thus, 48 treatment plans were established according to the three types of spine levels, four types of target shapes, two MLC leaf widths, and two types of planning techniques. The established treatment plans are shown in Figure 2.

Table 1 Target and spinal cord volumes

\begin{tabular}{lccccc}
\hline & \multicolumn{4}{c}{ Target (cc) } & $\begin{array}{c}\text { Spinal } \\
\text { cord (cc) }\end{array}$ \\
\cline { 2 - 5 } & Type 1 & Type 2 & Type 3 & Type 4 & cor \\
\hline C5 & 4.62 & 6.37 & 7.67 & 9.90 & 7.37 \\
T5 & 14.19 & 18.60 & 23.18 & 27.69 & 9.14 \\
L2 & 30.32 & 35.02 & 38.98 & 44.45 & 9.76 \\
\hline
\end{tabular}

\section{Dosimetric indices}

Target volume coverage (TVC), conformity index (CI), dose gradient index $(\mathrm{GI})$, and $\mathrm{V}_{30 \%}$ were used to compare the efficacy of each treatment plan. The definition of each index is summarized below.

1. TVC: The index to evaluate the dose coverage of the target volume $[25,26]$. A larger value of TVC indicates a better dose coverage of the target volume.

$$
\operatorname{TVC}(\%)=\frac{\begin{array}{l}
\text { Volume within the target receiving } \\
\text { at least the prescription isodose }
\end{array}}{\text { Target volume }} \times 100(\%)
$$

2. CI: The ratio used to evaluate the quality of fit of the target volume to the prescription isodose volume. It was proposed by the Radiation Therapy Oncology Group (RTOG) and modified by Paddick et al. [27] and Nakamura et al. [28]. A smaller value of CI indicates a better conformity of the target volume.

$$
\text { Conformity index }(\mathrm{CI})=\frac{\mathrm{PIV} \times \mathrm{TV}}{\mathrm{PTV}_{\mathrm{PIS}} \times \mathrm{PTV}_{\mathrm{PIS}}}
$$



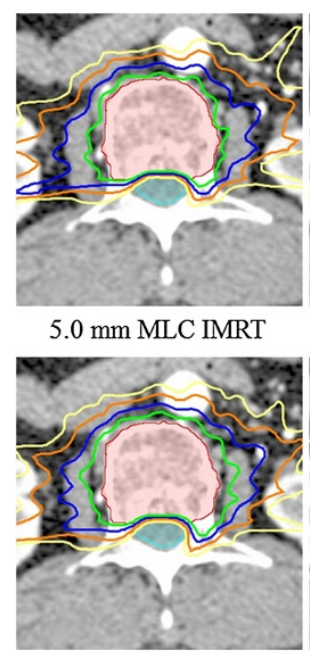

$2.5 \mathrm{~mm}$ MLC IMRT

(a) Type 1

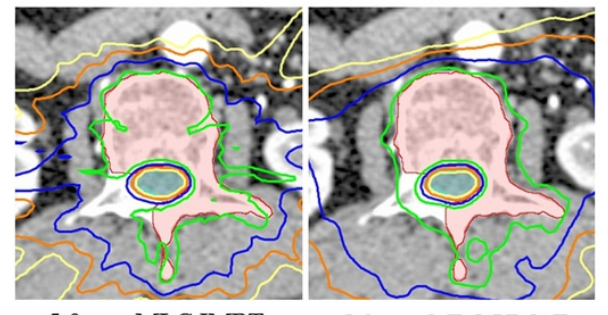

$5.0 \mathrm{~mm}$ MLC IMRT

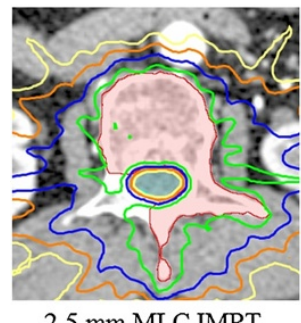

$2.5 \mathrm{~mm}$ MLC IMRT

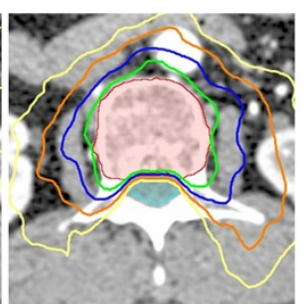

$5.0 \mathrm{~mm}$ MLC VMAT

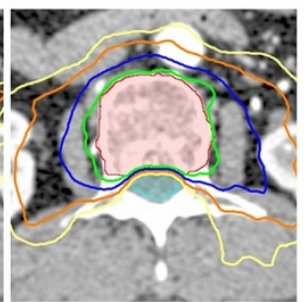

$2.5 \mathrm{~mm}$ MLC VMAT

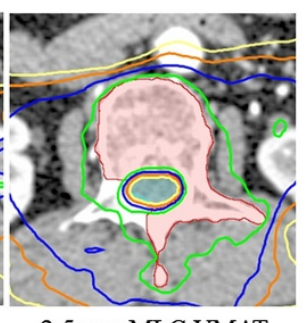

(c) Type 3

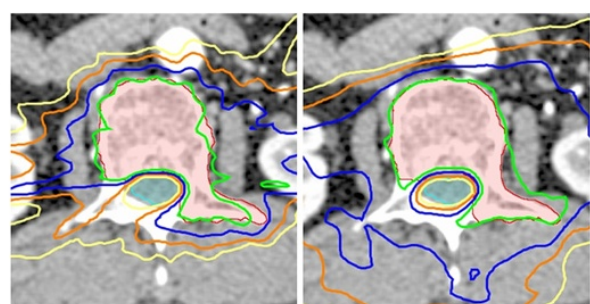

$5.0 \mathrm{~mm}$ MLC VMAT

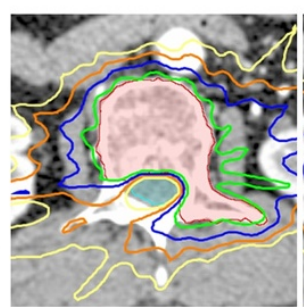

$2.5 \mathrm{~mm}$ MLC IMRT

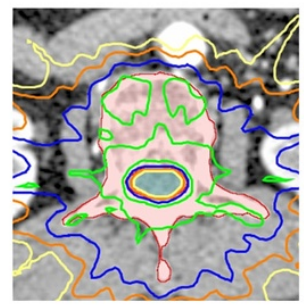

$5.0 \mathrm{~mm}$ MLC IMRT

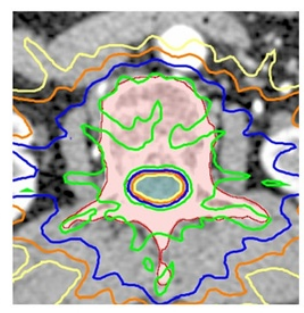

$2.5 \mathrm{~mm}$ MLC IMRT (b) Type 2

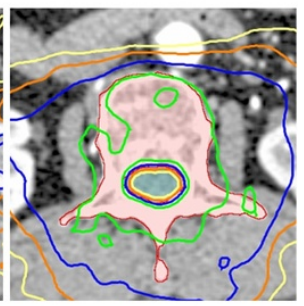

$5.0 \mathrm{~mm}$ MLC VMAT
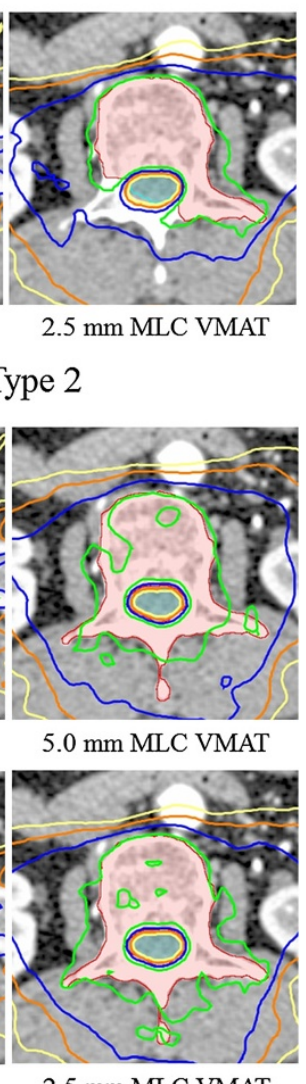

(d) Type 4

Figure 2 Established treatment plans according to the four types of target shapes, two MLC leaf widths, and two types of planning techniques. (a) Type 1, (b) Type 2, (c) Type 3, and (d) Type 4 (the green line is the 18 Gy isodose line, blue line is the 15 Gy isodose line, orange line is the $12 \mathrm{~Gy}$ isodose line, yellow line is the $10 \mathrm{~Gy}$ isodose line).

[PIV, prescription isodose volume; PTV $\mathrm{PIS}$, planning target volume encompassed within the prescription isodose surface; TV, target volume]

3. GI: The index that represent the degree of dose drop-off outside the target volume, which was proposed by Paddick et al. [29]. A smaller value of GI indicates a better degree of dose drop-off outside the target volume.

Dose gradient index $(\mathrm{GI})=\frac{V_{50}}{P T V_{\mathrm{PIS}}} \times 100(\%)$
$\left[\mathrm{V}_{50}\right.$, volume receiving at least $50 \%$ dose of the pre-

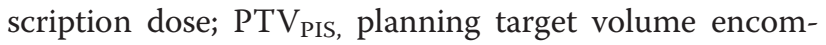
passed within the prescription isodose surface]

4. Improvement ratio: The ratio used to evaluate the improvement in the index between the two rival plans (a plan with a $2.5-\mathrm{mm} \mathrm{MLC} \mathrm{vs.} \mathrm{a} \mathrm{plan} \mathrm{with} \mathrm{a} 5-\mathrm{mm}$ MLC) [30].

$$
\begin{aligned}
\operatorname{Improvement} \text { ratio }(\%)= & \frac{\left(\text { Index }_{2.5-\mathrm{mm} \mathrm{MLC}}-\text { Index }_{5-\mathrm{mm} \mathrm{MLC}}\right)}{\operatorname{Index} 5-\mathrm{mm} \mathrm{MLC}} \\
& \times 100(\%)
\end{aligned}
$$


5. $\mathrm{V}_{30 \%}$ : The irradiated volume receiving more than $30 \%$ of the prescription dose.

\section{Statistical analysis}

An independent $t$-test was used to analyze the influence of the MLC size, and the difference according to the types of target shapes was evaluated using a one-way analysis of variance test. A Wilcoxon signed rank test was used to compare the low dose distribution $\left(\mathrm{V}_{30 \%}\right)$ between IMRT and VMAT plans according to the MLC leaf width and the types of target. The statistical analysis was conducted using MedCalc version 12.6 for Windows (MedCalc Software, Ostend, Belgium) and a $p$ value $<0.05$ was considered statistically significant.

\section{Results}

Comparison between 2.5- $\mathrm{mm}$ and 5-mm MLCs in IMRT and VMAT plans

The dosimetric indices and their improvement ratios according to the MLC leaf width $(2.5 \mathrm{~mm}$ vs. $5 \mathrm{~mm})$ and modulated techniques (IMRT vs. VMAT) are summarized in Table 2. The $\mathrm{D}_{0.25 \mathrm{cc}}$ of the spinal cord was a median of 10.00 Gy (range: 9.97-10.02 Gy), which was not statistically different in relation to the MLC type, planning technique (IMRT vs. VMAT), types of target shapes, and spine level $(p=0.087,0.087,0.994$, and 0.471 , respectively).

The mean TVC was $88.10 \%$ with the 5 -mm MLC and 91.83\% with the $2.5-\mathrm{mm}$ MLC. When using the $2.5-\mathrm{mm}$ MLC instead of the 5-mm MLC, TVC was improved by $5.68 \%$, which was statistically significant $(p=0.003)$. In IMRT, the mean TVC was $83.55 \%$ with the 5 - mm MLC and $88.40 \%$ with the 2.5 - $\mathrm{mm}$ MLC, and the improvement ratio was $8.38 \%$. In VMAT, the mean TVC was 92.65\% with the $5-\mathrm{mm} \mathrm{MLC}$ and $95.26 \%$ with the $2.5-\mathrm{mm}$ MLC, and the improvement ratio was $2.97 \%$. TVC was higher in VMAT than in IMRT, and the improvement ratio was higher in IMRT (Figure 3).
The mean CI was 2.06 with the 5-mm MLC and 1.94 with the $2.5-\mathrm{mm}$ MLC; this difference is not statistically significant $(p=0.110)$. In the case of IMRT, the mean CI was 2.24 with the $5-\mathrm{mm}$ and 2.24 with the $2.5-\mathrm{mm}$ MLC. In the case of VMAT, the mean CI was 1.88 with the $5-\mathrm{mm}$ and 1.85 with the $2.5-\mathrm{mm}$ MLC. There is no statistically significant difference between the MLC leaf widths ( $p=0.042$ and 0.689 , respectively). CI was larger in VMAT than in IMRT.

The mean GI was 10.89 with the 5-mm MLC and 9.99 with the 2.5-mm MLC. Comparing the 2.5- $\mathrm{mm}$ with the 5 -mm MLC, GI was improved by $6.25 \%$, which is statistically significant $(p=0.023)$. In IMRT, the mean $\mathrm{CI}$ was 10.98 and 9.30 with the $5-\mathrm{mm}$ and the $2.5-\mathrm{mm}$ MLC, respectively, and the improvement ratio was $13.79 \%$, which is statistically significant $(p=0.003)$. In VMAT, the mean GI was 10.80 and 10.68 with the $5-\mathrm{mm}$ and the $2.5-\mathrm{mm}$ MLC, respectively, and the difference is not statistically significant $(p=0.871)$.

\section{Comparison of $\mathrm{V}_{30 \%}$ between IMRT and VMAT plans} according to the MLC leaf widths and the types of target To evaluate the low dose distribution of the IMRT and VMAT plans, the $\mathrm{V}_{30 \%}$ values are compared according to the MLC leaf widths and the types of target (Table 3 ). The mean $\mathrm{V}_{30 \%}$ was $460.80 \mathrm{~cm}^{3}$ and $466.44 \mathrm{~cm}^{3}$ in the IMRT and VMAT plans, respectively; the difference is not statistically significant $(p=1.000)$.

In the $2.5-\mathrm{mm}$ MLC, the mean $\mathrm{V}_{30 \%}$ was $455.84 \mathrm{~cm}^{3}$ and $466.10 \mathrm{~cm}^{3}$ in the IMRT and VMAT plans, respectively. In the $5.0-\mathrm{mm}$ MLC, the mean $\mathrm{V}_{30 \%}$ was $465.76 \mathrm{~cm}^{3}$ and $466.78 \mathrm{~cm}^{3}$ in the IMRT and VMAT plans, respectively. In both types of MLC, there was no statistically significant difference $(p=0.875$ for 2.5 - $\mathrm{mm}$ and 0.875 for 5.0-mm MLC).

According to the types of target, the mean $\mathrm{V}_{30 \%}$ for IMRT and VMAT were, respectively, $315.33 \mathrm{~cm}^{3}$ and $380.36 \mathrm{~cm}^{3}$ for type $1,439.84 \mathrm{~cm}^{3}$ and $470.90 \mathrm{~cm}^{3}$ for type $2,551.47 \mathrm{~cm}^{3}$ and $514.65 \mathrm{~cm}^{3}$ for type 3 , and $536.55 \mathrm{~cm}^{3}$

Table 2 Dosimetric indices and their improvement ratios according to the MLC leaf width (2.5 $\mathrm{mm}$ vs. $5 \mathrm{~mm}$ ) and intensity-modulated techniques (IMRT vs. VMAT)

\begin{tabular}{|c|c|c|c|c|c|}
\hline & & 2.5-mm MLC & 5-mm MLC & Improvement ratio (\%) & $p$ value \\
\hline \multirow[t]{3}{*}{ Overall } & TVC & $91.83 \pm 11.56$ & $88.10 \pm 15.23$ & $5.68 \pm 10.07$ & 0.003 \\
\hline & $\mathrm{Cl}$ & $1.94 \pm 0.53$ & $2.06 \pm 0.81$ & $-2.42 \pm 12.25$ & 0.110 \\
\hline & $\mathrm{Gl}$ & $9.99 \pm 2.13$ & $10.89 \pm 2.81$ & $-6.25 \pm 18.84$ & 0.023 \\
\hline \multirow[t]{3}{*}{ IMRT } & TVC & $88.40 \pm 15.62$ & $83.55 \pm 20.24$ & $8.38 \pm 13.66$ & 0.042 \\
\hline & $\mathrm{Cl}$ & $2.03 \pm 0.67$ & $2.24 \pm 1.06$ & $-4.86 \pm 13.00$ & 0.119 \\
\hline & $\mathrm{Gl}$ & $9.30 \pm 2.06$ & $10.98 \pm 3.34$ & $-13.79 \pm 7.38$ & 0.003 \\
\hline \multirow[t]{3}{*}{ VMAT } & TVC & $95.26 \pm 3.12$ & $92.65 \pm 5.48$ & $2.97 \pm 3.10$ & 0.005 \\
\hline & $\mathrm{Cl}$ & $1.85 \pm 0.34$ & $1.88 \pm 0.41$ & $0.02 \pm 11.48$ & 0.689 \\
\hline & Gl & $10.68 \pm 2.04$ & $10.80 \pm 2.30$ & $1.27 \pm 23.74$ & 0.871 \\
\hline
\end{tabular}




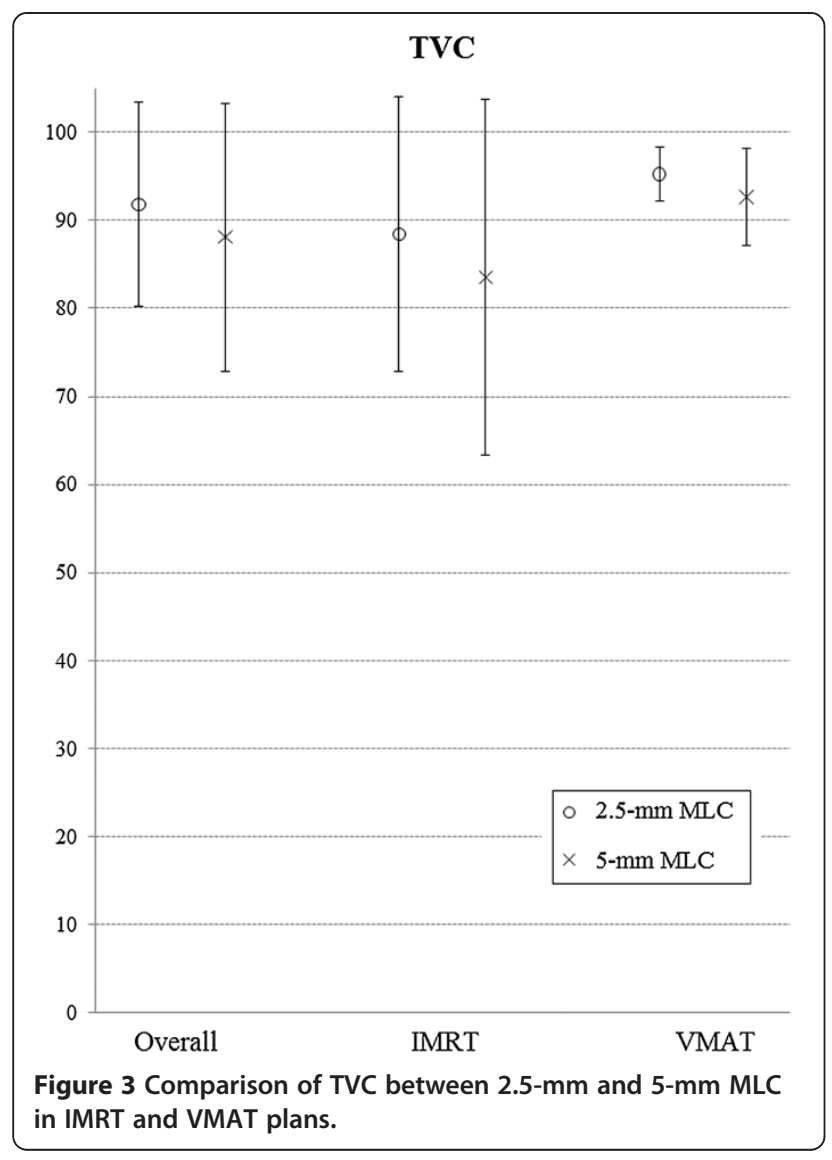

and $499.86 \mathrm{~cm}^{3}$ for type 4 . For all target types, there was no statistically significant difference $(p=0.075,0.173$, 0.249 , and 0.249, respectively).

\section{Improvement ratio according to the types of target}

The improvement ratios (the 2.5-mm MLC compared to the 5-mm MLC) of the dosimetric indices according to the types of target shapes in IMRT and VMAT are summarized in Table 4.

The mean improvement ratio of TVC was $0.42 \%$ in type $1,2.39 \%$ in type $2,2.74 \%$ in type 3 , and $17.16 \%$ in type 4 . There was a statistically significant difference in

Table 3 Comparison of $\mathbf{V}_{\mathbf{3 0}}$ between IMRT and VMAT plans according to the MLC leaf width and the types of target

\begin{tabular}{ccccc}
\hline & & IMRT $\left(\mathbf{c m}^{\mathbf{3}}\right)$ & VMAT $\left(\mathbf{c m}^{\mathbf{3}}\right)$ & $\boldsymbol{p}$ value \\
\hline MLC & 2.5-mm MLC & $455.84 \pm 316.45$ & $466.10 \pm 288.69$ & 0.875 \\
\multirow{4}{*}{ Target shapes } & 5.0-mm MLC & $465.76 \pm 315.02$ & $466.78 \pm 276.48$ & 0.875 \\
& Type 1 & $315.33 \pm 216.79$ & $380.36 \pm 257.99$ & 0.075 \\
& Type 2 & $439.84 \pm 287.24$ & $470.90 \pm 290.22$ & 0.173 \\
& Type 3 & $551.47 \pm 368.23$ & $514.65 \pm 305.96$ & 0.249 \\
Overall & Type 4 & $536.55 \pm 361.91$ & $499.86 \pm 296.27$ & 0.249 \\
\hline
\end{tabular}

type 4 compared with other types $(1,2$, and 3$)(p=0.006)$, and the difference in the improvement ratio was $14.42-$ 16.74\%. In IMRT, the improvement ratios of Type 1, 2, 3, and 4 were $-0.16 \%, 3.62 \%, 2.10 \%$, and $27.99 \%$, respectively. In VMAT, the improvement ratios for types 1, 2, 3, and 4 were $1.01 \%, 1.15 \%, 3.37 \%$, and $6.34 \%$, respectively (Figure 4). A statistical difference was observed only in IMRT $(p=0.007)$.

The mean improvement ratio of CI was $-0.41 \%$ in type $1,-5.58 \%$ in type $2,3.77 \%$ in type 3 , and $-7.76 \%$ in type 4. However, there was no statistically significant difference between different target shapes $(p=0.396)$, and there was no statistically significant difference between IMRT and VMAT ( $p=0.231$ and 0.883 , respectively).

The mean improvement ratio of GI was $8.89 \%$ in type $1,-10.61 \%$ in type $2,-7.85 \%$ in type 3 , and $-15.46 \%$ in type 4 . However, there was no statistically significant difference $(p=0.122)$. In IMRT, the mean improvement ratios of types $1,2,3$, and 4 were $-8.85 \%,-14.30 \%$, $-9.37 \%$, and $-22.62 \%$, respectively, and a statistically significant difference was observed $(p=0.047)$. However, there was no statistically significant difference in VMAT $(p=0.208)$.

\section{Discussion}

Owing to developments in MLCs in addition to improvements in radiotherapy planning techniques and delivery methods, radiosurgery has been widely used, and many studies have reported its dosimetric superiority and clinical effectiveness [3,5-8]. Jin et al. reported that when a dose of 18 Gy was prescribed, and $10 \%$ of the adjacent spinal cord volume was irradiated below $10 \mathrm{~Gy}, 85 \%$ of patients safely experienced pain relief and neurological improvement [4].

The micro-MLC commonly used for radiosurgery is defined as having a leaf width of less than $5 \mathrm{~mm}$. Galal et al. tested the dosimetric and mechanical characteristics of a 3-mm micro-MLC and concluded that it was suitable for use in radiosurgery [31]. Many investigators have studied the effects of MLC leaf width in various planning techniques (3D-CRT and IMRT). Monk et al. compared 3-mm and 5-mm MLC in radiosurgery with the 3D-CRT technique for 14 brain lesions [13]. CI was better with the 3-mm MLC, but there was no statistically significant improvement in the index for organs at risk ( $D_{\max }$ to the critical structures). Kubo et al. found, while studying the difference of MLC size in radiosurgery with 3D-CRT for prostate cancer, that when a 10-mm MLC was replaced with a 3-mm MLC, the dose to the bladder and rectum was lowered, but CI was not improved [17]. Wu et al. compared IMRT plans using 5-mm and 2.5-mm MLCs for 15 cases of brain, liver, and spine lesions. In the brain lesion, the indices for target volume such as $D_{\min }, D_{\max }$, and the homogeneity 
Table 4 Improvement ratios of the dosimetric indices according to the types of target in IMRT and VMAT

\begin{tabular}{|c|c|c|c|c|c|c|}
\hline & & \multicolumn{5}{|c|}{ Improvement ratio (\%) } \\
\hline & & Type 1 & Type 2 & Type 3 & Type 4 & $p$ value \\
\hline \multirow[t]{3}{*}{ Overall } & TVC & $0.42 \pm 1.01$ & $2.39 \pm 5.24$ & $2.74 \pm 2.80$ & $17.16 \pm 14.68$ & 0.006 \\
\hline & $\mathrm{Cl}$ & $-0.41 \pm 9.34$ & $-5.58 \pm 6.61$ & $3.77 \pm 9.00$ & $-7.46 \pm 19.63$ & 0.396 \\
\hline & $\mathrm{Gl}$ & $8.89 \pm 33.04$ & $-10.61 \pm 4.40$ & $-7.85 \pm 5.31$ & $-15.46 \pm 9.52$ & 0.122 \\
\hline \multirow[t]{3}{*}{ IMRT } & TVC & $-0.16 \pm 0.36$ & $3.62 \pm 7.97$ & $2.10 \pm 2.32$ & $27.99 \pm 13.35$ & 0.007 \\
\hline & $\mathrm{Cl}$ & $-4.97 \pm 8.94$ & $-6.83 \pm 4.75$ & $7.16 \pm 9.91$ & $-14.81 \pm 18.92$ & 0.231 \\
\hline & $\mathrm{Gl}$ & $-8.85 \pm 4.59$ & $-14.30 \pm 2.43$ & $-9.37 \pm 5.68$ & $-22.62 \pm 7.57$ & 0.047 \\
\hline \multirow[t]{3}{*}{ VMAT } & TVC & $1.01 \pm 1.18$ & $1.15 \pm 0.78$ & $3.37 \pm 3.61$ & $6.34 \pm 3.11$ & 0.094 \\
\hline & $\mathrm{Cl}$ & $4.14 \pm 8.72$ & $-4.33 \pm 9.06$ & $-0.39 \pm 8.35$ & $-0.11 \pm 21.06$ & 0.883 \\
\hline & $\mathrm{Gl}$ & $26.64 \pm 41.98$ & $-6.92 \pm 1.32$ & $-6.33 \pm 5.59$ & $-8.29 \pm 3.92$ & 0.208 \\
\hline
\end{tabular}

index $(\mathrm{HI})$ were improved, and in the spine lesion, the indices for organs at risk such as $\mathrm{D}_{1}, \mathrm{D}_{10}$, and the mean dose to the cord were improved by $14 \%, 19 \%$, and $29 \%$, respectively [16]. Wang et al. compared IMRT plans using $10-\mathrm{mm}$ and 4-mm MLCs for 10 patients with prostate cancer, and both the indices for target volume $\left(D_{\max -1 \mathrm{cc}}\right.$, $\mathrm{D}_{99}$, and $\mathrm{HI}$ ), and dose to the rectum and bladder were improved significantly [32,33]. According to Jin et al. and Dvorack et al., CI and TVC were improved significantly when the MLC leaf width was changed from $10 \mathrm{~mm}$ to

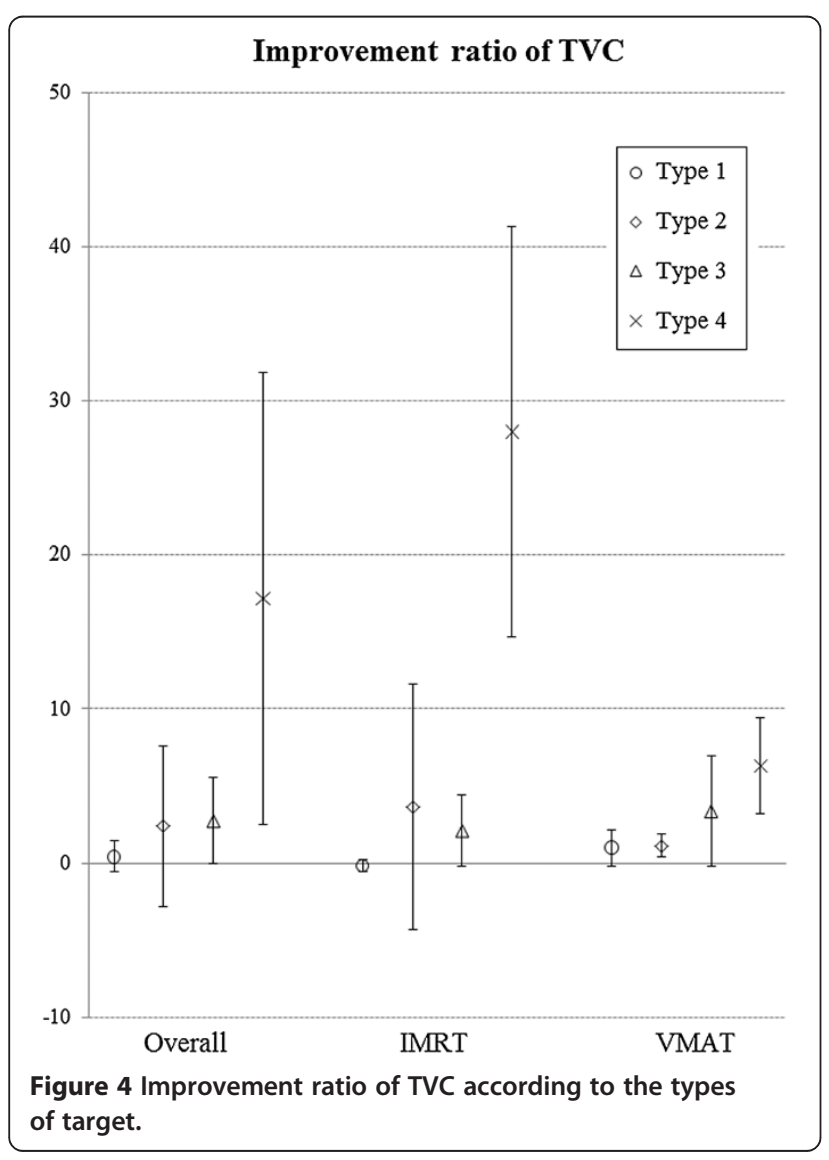

$3 \mathrm{~mm}$ in the IMRT plans [14,34]. However, according to Burmeister et al., when they compared a $10-\mathrm{mm}$ MLC with a 5-mm MLC in the IMRT plans, there were no significant differences in $\mathrm{D}_{\min }, \mathrm{D}_{\max }$, and $\mathrm{D}_{\text {mean }}$. The treatment efficacy was lower for the 5-mm MLC because of an increase in the whole body dose, the treatment time, and the monitor unit [35].

These effects of the MLC leaf width varied according to the radiotherapy technique used. The benefits of a small MLC were more prominent in the 3D-CRT compared to the IMRT technique $[14,34,36]$. Tanyi et al. analyzed the impact of the MLC leaf width difference ( $2.5 \mathrm{~mm}$ vs. $5 \mathrm{~mm}$ ) on the 3D-CRT, DCAT, and IMRT techniques for 68 cases of brain lesions. In DCAT, the indices for target volume $\left(\mathrm{CI}, \mathrm{D}_{\min }, \mathrm{D}_{\max }\right.$, and $\left.\mathrm{D}_{\text {mean }}\right)$ and index for organs at risk (PRV100, peritumoral rind volume receiving $\geq 100 \%$ of the prescription dose) were improved, while in 3D-CRT, only the indices for target volume $\left(\mathrm{CI}\right.$ and $\left.\mathrm{D}_{\mathrm{min}}\right)$ were improved. However, in IMRT, there was no improvement in the indices for target volume $\left(\mathrm{CI}, \mathrm{D}_{\min }, \mathrm{D}_{\max }\right.$, and $\left.\mathrm{D}_{\text {mean }}\right)$ and organs at risk (PRV100) [36].

Recently, the VMAT technique was developed by combining IMRT with the concept of dynamic arc therapy, and many authors have reported results comparing static IMRT and VMAT [2,3,24,37]. The studies of 7-field IMRT compared with VMAT showed that 1-arc VMAT was similar to or slightly more effective than IMRT $[2,37]$. Lee et al. compared 7-field IMRT and 1-arc VMAT plans for 5 cases of spine lesions and reported that 1-arc VMAT was better than 7-field IMRT in CI and $\mathrm{D}_{90}$, and the dose to the spinal cord was significantly lower in 1-arc VMAT $(p=0.04)$ [2]. Tsai et al. compared 7-field IMRT with 1-arc VMAT for 12 patients with prostate cancer, and 1-arc VMAT showed better result than 7-field IMRT both in Nakamura's CI and dose to the rectum [37]. Reports have shown 2-arc VMAT to be superior to IMRT, but if the number of fields is increased in IMRT, it has been reported to be better than 1-arc VMAT and similar to 2-arc 
VMAT [3,24]. Roa et al. compared 7-14 fields IMRT, 1-arc VMAT, and 2-arc VMAT plan for 23 cases of brain and body lesions, and there were no statistically significant differences among the three groups according to the RTOG CI, Nakamura's CI, and HI [24]. Wu et al. reported the results from comparing 8-12 fields IMRT, 1-arc VMAT, and 2-arc VMAT plans for 10 cases of spine lesions. Paddick's CI was only improved in 2-arc VMAT compared to 8-12 fields IMRT, and all other comparison indices $\left(\mathrm{D}_{99}, \mathrm{D}_{95}, \mathrm{D}_{10}, \mathrm{D}_{5}, \mathrm{D}_{1}\right.$, and $\left.\mathrm{D}_{\text {mean }}\right)$ showed no statistically significant difference [3].

The basic concept of radiation therapy planning is the balancing of two opposing objectives: the target volume should be irradiated with as much of the prescribed dose as possible, and organs at risk should be spared from radiation as much as possible. Therefore, there are limitations to improve the dose coverage to the target volume while sparing the organs at risk. Among previously published studies, only a few reported that the indices for target volume and organs at risk could be improved simultaneously $[18,32]$. Other authors have reported the improvement of only one type of index [13-15,17].

In this study, all the treatment plans were designed to obtain the maximal target volume coverage satisfying the dose constraint of the spinal cord (maximum 10 Gy to $0.25 \mathrm{cc}$ of spinal cord). Therefore, the irradiated dose to $0.25 \mathrm{cc}$ of spinal cord was the same in all treatment plans. The present study was designed mainly to enable comparison of the indices for target volume, and thus, the comparison of the index for the spinal cord, which is the most important organ at risk in the treatment of the spinal lesions, was not necessary. In addition, to enhance the quality of the IMRT and VMAT plans, 11-field IMRT and 3-arc VMAT plans were carried out.

In this study, when the 5-mm MLC was replaced by the 2.5-mm MLC, TVC and GI improved significantly by $5.68 \%$ and $6.25 \%$, respectively. With a smaller MLC leaf width, the improvement ratios of TVC were larger in IMRT than in VMAT (8.38\% vs. $2.97 \%)$. Although the improvement was prominent in IMRT, the values of TVC were better in VMAT compared to IMRT. According to previous studies, 3D-CRT and DCAT are more sensitive to the MLC leaf width than IMRT $[14,34,36]$. We think that more sophisticated radiotherapy techniques cause higher dosimetric index values. Such techniques are less affected by the MLC leaf width. These trends were observed in our study as well.

The VMAT technique has wider low-dose regions than IMRT [38,39]. However, some authors have reported that VMAT does not always increase the regions of lowdose distribution compared to IMRT $[2,40,41]$. Lee et al. compared 7-field IMRT and 1-arc VMAT for spine metastases [2]. When prescribing $35 \mathrm{~Gy}$, the $\mathrm{V}_{10 \mathrm{~Gy}}$ for the remaining volume at risk was larger in IMRT than in
VMAT $(14.6 \%$ vs. $11 \%)$, which was statistically significant $(p=0.04)$. According to Wolff et al., in their study comparing VMAT and IMRT, $\mathrm{V}_{30 \%}$ was higher in 7-field IMRT than in 2-arc VMAT $\left(3,414 \mathrm{~cm}^{3}\right.$ vs. $\left.3,340 \mathrm{~cm}^{3}\right)$ [41]. However, in the case of 1 -arc VMAT, $V_{30 \%}$ was $3,438 \mathrm{~cm}^{3}$, which was higher than that in 7-field IMRT. In this study, $\mathrm{V}_{30 \%}$ was $460.80 \mathrm{~cm}^{3}$ in IMRT and $466.44 \mathrm{~cm}^{3}$ in VMAT, the difference of which is not statistically significant $(p=1.000)$. Lee et al. reported that the health tissue mean dose was larger in 18-field IMRT than in 7-field IMRT (29.37 Gy vs. 29.11 Gy) [42]. Therefore, the regions of low-dose distribution could differ on the basis of the number of fields and the type of parameter.

Several studies have reported that the micro-MLC and IMRT techniques are more effective when the target is small or its shape is complex [14,16-18,36]. According to the study of Kubo et al. on the effects of the MLC leaf width difference $(1.7 \mathrm{~mm}, 3 \mathrm{~mm}$, and $10 \mathrm{~mm})$, when target shapes became more irregular, a smaller-width MLC leaf was more efficient [17]. Dhabaan et al. investigated the effect of the MLC leaf width difference $(2.5 \mathrm{~mm}$ vs. $5 \mathrm{~mm}$ ) and reported that when the target became small and had a complex shape, CI improved and the indices for organs at risk such as the conformity distance index, NTV50\% (normal tissue volume $\geq 50 \%$ of prescription dose), NTV70\%, and NTV90\% also improved [18]. However, these researches were limited because the target complexities were not methodically classified.

In this study, the complexities of the target were classified into four types by using the WBB surgical staging system. The effects of the MLC leaf width were evaluated according to the type of target. Our study showed that a smaller MLC leaf width enhanced the target coverage. The improvement ratio of TVC in type 4, which was the most complex target shape, was $17.16 \%$, and TVC was improved by $14.42-16.74 \%$ in type 4 compared to other types of targets. This means that as the target became more irregular, the dosimetric advantage of the smaller MLC increased. When a 2.5-mm MLC was used in VMAT, TVC was improved by only $6.34 \%$ in type 4 , which was not statistically significant $(p=0.094)$. However, in IMRT, TVC was improved by $27.99 \%$ in Type 4 , and the difference between type 4 and types $1-3$ was $24.37-28.15 \%$, which is statistically significant $(p=0.007)$. In addition, GI was significantly improved in IMRT $(p=0.047)$.

In conclusion, a smaller MLC leaf width provided improved target coverage in both IMRT and VMAT, and its improvement was larger in IMRT than in VMAT. In addition, a smaller MLC leaf width was more effective for a complex-shaped target.

\section{Competing interests}

The authors declare that they have no competing interests. 


\section{Authors' contributions}

SMC and SHS designed the experiment, and SMC performed the data collection. GWL performed the treatment planning and conducted all planning evaluations. SMC and SHS interpreted the data as well. SHS performed the statistical analysis. SMC drafted the manuscript. All authors have read and approved the final manuscript.

\section{Author details}

'Department of Radiation Oncology, Cheju Halla General Hospital, Jeju, Korea. ${ }^{2}$ Department of Radiation Oncology, Incheon St. Mary's hospital, College of Medicine, the Catholic University of Korea, Incheon, Korea.

Received: 18 June 2013 Accepted: 2 March 2014

Published: 8 March 2014

\section{References}

1. Schuster JM, Grady MS: Medical management and adjuvant therapies in spinal metastatic disease. Neurosurg Focus 2001, 11(6):e3.

2. Lee YK, Bedford JL, MCNair HA, Hawkins MA: Comparison of deliverable IMRT and VMAT for spine metastases using a simultaneous integrated boost. Br J Radiol 2013, 86(1022):20120466.

3. Wu QJ, Yoo S, Kirkpatrick JP, Thongphiew D, Yin FF: Volumetric arc intensity-modulated therapy for spine body radiotherapy: comparison with static intensity-modulated treatment. Int J Radiat Oncol Biol Phys 2009, 75(5):1596-1604.

4. Jin JY, Chen Q, Jin R, Rock J, Anderson J, Li S, Movsas B, Ryu S: Technical and clinical experience with spine radiosurgery: a new technology for management of localized spine metastases. Technol Cancer Res Treat 2007, 6(2):127-133.

5. Yin FF, Ryu S, Ajlouni M, Zhu J, Yan H, Guan H, Faber K, Rock J, Abdalhak M, Rogers L, Rosenblum M, Kim JH: A technique of intensity-modulated radiosurgery (IMRS) for spinal tumors. Med Phys 2002, 29(12):2815-2822.

6. Ryu S, Fang Yin F, Rock J, Zhu J, Chu A, Kagan E, Rogers L, Ajlouni M, Rosenblum M, Kim JH: Image-guided and intensity-modulated radiosurgery for patients with spinal metastasis. Cancer 2003, 97(8):2013-2018.

7. Shiu AS, Chang EL, Ye JS, Lii M, Rhines LD, Mendel E, Weinberg J, Singh S, Maor MH, Mohan R, Cox JD: Near simultaneous computed tomography image-guided stereotactic spinal radiotherapy: an emerging paradigm for achieving true stereotaxy. Int J Radiat Oncol Biol Phys 2003, 57(3):605-613.

8. Yin FF, Ryu S, Ajlouni M, Yan H, Jin JY, Lee SW, Kim J, Rock J, Rosenblum M, Kim JH: Image-guided procedures for intensity-modulated spinal radiosurgery. Technical note. J Neurosurg 2004, 101(3):419-424

9. Solberg TD, Boedeker KL, Fogg R, Selch MT, DeSalles AA: Dynamic arc radiosurgery field shaping: a comparison with static field conformal and noncoplanar circular arcs. Int J Radiat Oncol Biol Phys 2001, 49(5):1481-1491.

10. Benedict SH, Cardinale RM, Wu Q, Zwicker RD, Broaddus WC, Mohan R: Intensity-modulated stereotactic radiosurgery using dynamic micromultileaf collimation. Int J Radiat Oncol Biol Phys 2001, 50(3):751-758.

11. Otto K: Volumetric modulated arc therapy: IMRT in a single gantry arc. Med Phys 2008, 35(1):310-317.

12. Yu CX: Intensity-modulated arc therapy with dynamic multileaf collimation: an alternative to tomotherapy. Phys Med Biol 1995, 40(9):1435-1449.

13. Monk JE, Perks JR, Doughty D, Plowman PN: Comparison of a micromultileaf collimator with a 5-mm-leaf-width collimator for intracranial stereotactic radiotherapy. Int J Radiat Oncol Biol Phys 2003, 57(5):1443-1449.

14. Jin JY, Yin FF, Ryu S, Ajlouni M, Kim JH: Dosimetric study using different leaf-width MLCs for treatment planning of dynamic conformal arcs and intensity-modulated radiosurgery. Med Phys 2005, 32(2):405-411.

15. Tanyi JA, Summers PA, McCracken $C L$, Chen Y, Ku LC, Fuss M: Implications of a high-definition multileaf collimator (HD-MLC) on treatment planning techniques for stereotactic body radiation therapy (SBRT): a planning study. Radiat Oncol 2009, 4:22.

16. Wu QJ, Wang Z, Kirkpatrick JP, Chang Z, Meyer JJ, Lu M, Huntzinger C, Yin FF: Impact of collimator leaf width and treatment technique on stereotactic radiosurgery and radiotherapy plans for intra- and extracranial lesions. Radiat Oncol 2009, 4:3.
17. Kubo HD, Wilder RB, Pappas CT: Impact of collimator leaf width on stereotactic radiosurgery and $3 \mathrm{D}$ conformal radiotherapy treatment plans. Int J Radiat Oncol Biol Phys 1999, 44(4):937-945.

18. Dhabaan A, Elder E, Schreibmann E, Crocker I, Curran WJ, Oyesiku NM, Shu HK, Fox T: Dosimetric performance of the new high-definition multileaf collimator for intracranial stereotactic radiosurgery. J Appl Clin Med Phys 2010, 11(3):3040

19. Tomita K, Kawahara N, Kobayashi T, Yoshida A, Murakami H, Akamaru T: Surgical strategy for spinal metastases. Spine 2001, 26(3):298-306.

20. Choi D, Crockard A, Bunger C, Harms J, Kawahara N, Mazel C, Melcher R, Tomita K: Review of metastatic spine tumour classification and indications for surgery: the consensus statement of the Global Spine Tumour Study Group. Eur Spine J 2010, 19(2):215-222.

21. Boriani $S$, Weinstein JN, Biagini R: Primary bone tumors of the spine. Terminology and surgical staging. Spine 1997, 22(9):1036-1044.

22. Timmerman RD: An overview of hypofractionation and introduction to this issue of seminars in radiation oncology. Semin Radiat Oncol 2008, 18(4):215-222

23. Pirzkall A, Carol MP, Pickett B, Xia P, Roach M 3rd, Verhey LJ: The effect of beam energy and number of fields on photon-based IMRT for deepseated targets. Int J Radiat Oncol Biol Phys 2002, 53(2):434-442.

24. Roa DE, Schiffner DC, Zhang J, Dietrich SN, Kuo JV, Wong J, Ramsinghani NS, Al-Ghazi MS: The use of RapidArc volumetric-modulated arc therapy to deliver stereotactic radiosurgery and stereotactic body radiotherapy to intracranial and extracranial targets. Med Dosim 2012, 37(3):257-264.

25. Sankaranarayanan V, Ganesan S, Oommen S, Padmanaban TK, Stumpf J, Ayyangar KM: Study on dosimetric parameters for stereotactic radiosurgery and intensity-modulated radiotherapy. Med Dosim 2003, 28(2):85-90

26. Lomax NJ, Scheib SG: Quantifying the degree of conformity in radiosurgery treatment planning. Int J Radiat Oncol Biol Phys 2003, 55(5):1409-1419.

27. Paddick I: A simple scoring ratio to index the conformity of radiosurgical treatment plans. Technical note. J Neurosurg 2000, 93(3):219-222

28. Nakamura JL, Verhey LJ, Smith V, Petti PL, Lamborn KR, Larson DA, Wara WM, McDermott MW, Sneed PK: Dose conformity of gamma knife radiosurgery and risk factors for complications. Int J Radiat Oncol Biol Phys 2001, 51(5):1313-1319.

29. Paddick I, Lippitz B: A simple dose gradient measurement tool to complement the conformity index. J Neurosurg 2006, 105(Suppl):194-201.

30. Lee TF, Chao PJ, Wang CY, Lan JH, Huang YJ, Hsu HC, Sung CC, Su TJ, Lian $\mathrm{SL}$, Fang FM: Dosimetric comparison of helical tomotherapy and dynamic conformal arc therapy in stereotactic radiosurgery for vestibular schwannomas. Med Dosim 2011, 36(1):62-70.

31. Galal MM, Keogh S, Khalil S: Dosimetric and mechanical characteristics of a commercial dynamic microMLC used in SRS. Med Phys 2011, 38(7):4225-4231.

32. Wang L, Movsas B, Jacob R, Fourkal E, Chen L, Price R, Feigenberg S, Konski A, Pollack A, Ma C: Stereotactic IMRT for prostate cancer: dosimetric impact of multileaf collimator leaf width in the treatment of prostate cancer with IMRT. J App/ Clin Med Phys 2004, 5(2):29-41.

33. Wang L, Hoban P, Paskalev K, Yang J, Li J, Chen L, Xiong W, Ma CC: Dosimetric advantage and clinical implication of a micro-multileaf collimator in the treatment of prostate with intensity-modulated radiotherapy. Med Dosim 2005, 30(2):97-103.

34. Dvorak P, Georg D, Bogner J, Kroupa B, Dieckmann K, Potter R: Impact of IMRT and leaf width on stereotactic body radiotherapy of liver and lung lesions. Int J Radiat Oncol Biol Phys 2005, 61(5):1572-1581.

35. Burmeister J, McDermott PN, Bossenberger T, Ben-Josef E, Levin K, Forman JD: Effect of MLC leaf width on the planning and delivery of SMLC IMRT using the CORVUS inverse treatment planning system. Med Phys 2004, 31(12):3187-3193.

36. Tanyi JA, Kato CM, Chen Y, Chen Z, Fuss M: Impact of the high-definition multileaf collimator on linear accelerator-based intracranial stereotactic radiosurgery. Br J Radiol 2011, 84(1003):629-638.

37. Tsai CL, Wu JK, Chao HL, Tsai YC, Cheng JC: Treatment and dosimetric advantages between VMAT, IMRT, and helical tomotherapy in prostate cancer. Med Dosim 2011, 36(3):264-271.

38. Yoo S, Wu QJ, Lee WR, Yin FF: Radiotherapy treatment plans with RapidArc for prostate cancer involving seminal vesicles and lymph nodes. Int J Radiat Oncol Biol Phys 2010, 76(3):935-942. 
39. Wiezorek T, Brachwitz T, Georg D, Blank E, Fotina I, Habl G, Kretschmer M, Lutters G, Salz H, Schubert K, Wagner D, Wendt TG: Rotational IMRT techniques compared to fixed gantry IMRT and tomotherapy: multiinstitutional planning study for head-and-neck cases. Radiat Oncol 2011, 6:20.

40. Cozzi L, Dinshaw KA, Shrivastava SK, Mahantshetty U, Engineer R, Deshpande DD, Jamema SV, Vanetti E, Clivio A, Nicolini G, Fogliata A: A treatment planning study comparing volumetric arc modulation with RapidArc and fixed field IMRT for cervix uteri radiotherapy. Radiother Oncol 2008, 89(2):180-191.

41. Wolff D, Stieler F, Welzel G, Lorenz F, Abo-Madyan Y, Mai S, Herskind C, Polednik M, Steil V, Wenz F, Lohr F: Volumetric modulated arc therapy (VMAT) vs. serial tomotherapy, step-and-shoot IMRT and 3D-conformal RT for treatment of prostate cancer. Radiother Oncol 2009, 93(2):226-233.

42. Lee TF, Ting HM, Chao PJ, Fang FM: Dual arc volumetric-modulated arc radiotherapy (VMAT) of nasopharyngeal carcinomas: a simultaneous integrated boost treatment plan comparison with intensity-modulated radiotherapies and single arc VMAT. Clin Oncol 2012, 24(3):196-207.

doi:10.1186/1748-717X-9-72

Cite this article as: Chae et al.: The effect of multileaf collimator leaf width on the radiosurgery planning for spine lesion treatment in terms of the modulated techniques and target complexity. Radiation Oncology 2014 9:72.

\section{Submit your next manuscript to BioMed Central and take full advantage of:}

- Convenient online submission

- Thorough peer review

- No space constraints or color figure charges

- Immediate publication on acceptance

- Inclusion in PubMed, CAS, Scopus and Google Scholar

- Research which is freely available for redistribution 